\title{
NEPHROTIC SYNDROME ASSOCIATED WITH HYDRONEPHROSIS AND UNILATERAL RENAL AGENESIS
}

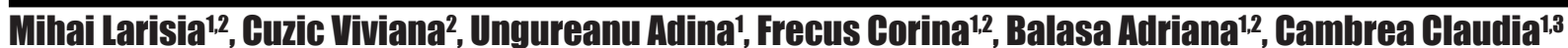

${ }^{1}$ Faculty of Medicine, University "Ovidius" of Constanta

${ }^{2}$ Pediatric Department, Clinical Emergency County Hospital of Constanta

${ }^{3}$ Clinical Hospital of Infectious Diseases of Constanta

\begin{abstract}
We reported a case of nephrotic syndrome in a child presenting undiagnosed hydronephrosis and unilateral renal agenesis in a 3 years and 9 months old male patient presenting features of nephrotic syndrome, with left hydronephrosis and unilateral renal agenesis. The association of nephrotic syndrome with congenital anomalies is extremely rare. The importance of this unusual combination was discussed respecting the literature.
\end{abstract}

Keywords: nephrotic syndrome, hydronephrosis, unilateral renal agenesis, childhood.

\section{Introduction}

URA (Unilateral renal agenesis) is defined as a congenital birth defect resulting an absence of the one-side renal tissue due to an abnormality of the embryonic kidney formation $(1,2)$.

URA may be often associated with congenital malformations of the contralateral kidney and/or urinary tract (3-5), like pelviureteric junction obstruction and vesicouretereal reflux.

Nephrotic syndrome is a disease affecting the glomeruli in which excessive amounts of protein are lost in urine. Excessive protein excretion typically lead to edema (accumulation of fluid in the body), low levels of albumin and high levels of fat in the blood (6).

\section{Gase presentation}

3 years and 9 months old male patient is admitted in the hospital for: swelling of the lower limbs and abdomen which were acute in onset, started 4 days ago and gradually progressing. The family haven't noticed other changes in the child's health condition.

\section{Clinical examination:}

- Afebrile, poor clinical status;

- Weight: $14 \mathrm{~kg}$ (10 \% percentile), Height: $89 \mathrm{~cm}$ (50\% percentile);

- Paleness; 
- “Pitting" edema of the lower limbs and lower abdomen, and also discrete edema of the face (Figure 1, Figure 2);

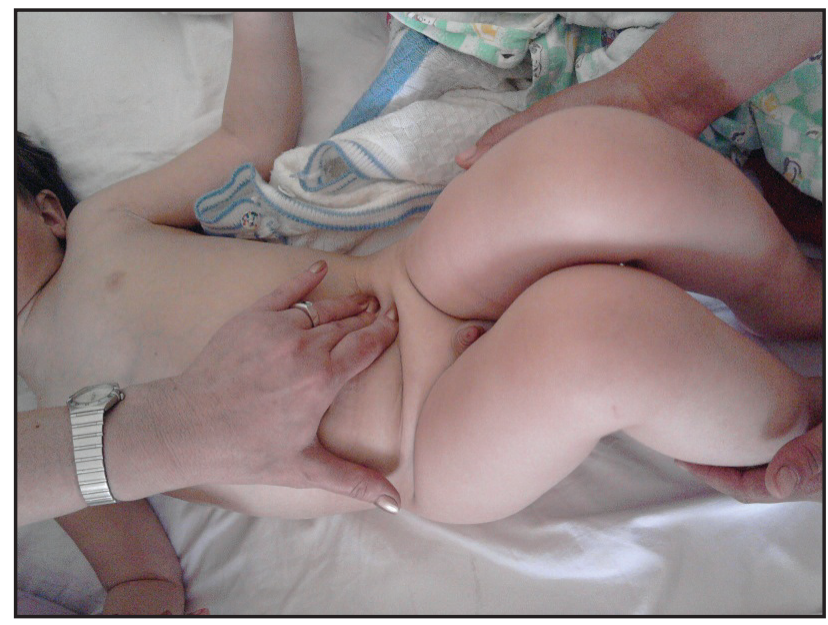

Figure 1. Edema of the lower abdomen

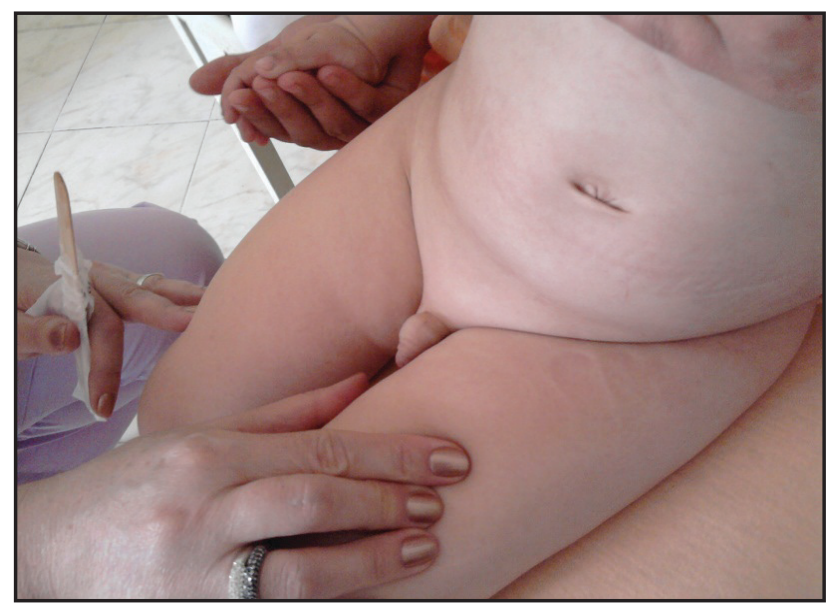

Figure 2. Edema of the lower limbs

- Diminished lung sounds and dullness on both sides, rare coughing;

- Pulse $=94 / \mathrm{min}$, regular, $\mathrm{BP}=110 / 80$ $\mathrm{mmHg}(50 \%$ percentile, age,weight,sex appropriate to normal range);

- Loss of appetite, distended abdomen, central umbilicus, umbilical scar in tension;

- Penile and scrotal edema;

- Low urinary output, normally colored, frothy urine.

Emergency laboratory tests showed:

- Massive proteinuria;

- Hypoproteinemia;

- Hypoalbuminaemia;

- Elevated erythrocyte sedimentation rate (ESR);
- Hypocalcemia;

Also, the sonography shows fluid accumulation in the peritoneal and pleural cavities leading to the hypotesis of Nephrotic Syndrome, requiring broadening the scope of investigations for positive and differential diagnosis.

Abdominal echography shows fluid accumulation in the abdominal cavity; the right kidney cannot be visualised in the right retroperitoneal space, also the left kidney with stage III hydronephrosis.

- Positive diagnosis:

- Idiopathic nephrotic syndrome ;

- Unilateral renal agenesis;

- Stage III- left kidney hydronephrosis.

After ruling out tuberculosis and hepatitis $\mathrm{B}$, the patient was started on steroids in the form of oral Prednisone at a dose of $60 \mathrm{mg} / \mathrm{m} \mathrm{2/}$ day. Also, the pacient received: substitution treatment with Human Albumin $0,5 \mathrm{~g} / \mathrm{kg}$ per day, intravenous; diuretic treatment with a loop diuretic, with strong diuretic effect (Furosemide) and potassium-sparing diuretics (Spironolactone) which worked more gently than the loop diuretics; antihypertensive and antiproteinuric treatment with ACE-inhibitors (Enalapril); Calcium, Potassium, vitamin D and Omega 3 (Polyunsaturated fatty acids) oral supplements; adequate hydration; low-sodium diet.

The pacient had a favorable outcome, both clinical and paraclinical, with the dissapearace of the edemas and the hypertension, the urinary output increased, losing $1,5 \mathrm{~kg}$ ( $10 \%$ from the initial weight); the appetite improves. After 12 days of treatment, the patient went into remission.

Subsequent echography shows no fluid in the peritoneal and pleural cavities, but the suspicion of renal agenesy with hydronephrosis is maintained.

Oral steroids were continued, in accordance with international protocols, for 4 weeks, daily, and then another 8 weeks, alternate days, with gradual decrease of the dosage, until treatment discontinuation. Adjuvant treatment was associated and it is recommended. The patient was followed-up regularly in our department and he remained in remission.

Health assesment after a year, with clinical, paraclinical and imaging parametres, showed a favorable output, with no relapses, no proteinuria, 
but the ultrasound result is maintaining the suspicion of right renal agenesy and stage III left kidney hydronephrosis.

A CT Urography (CTU) is required to confirm the renal malformation, but the pacient hasn't presented at the hospital.

8 years later, the pacient, 12 years old from now, is admitted in the Paediatric Clinic for assessment.

$\mathrm{He}$ is referred with the suspicion of right renal agenesy, stage III left kidney hydronephrosis, in a Paediatric Surgery clinic.

For establishing a proper diagnosis, the following investigations were made:

Blood and urine biochemistry showed normal range;

Kidney MRI Scan: Unique left renal structure. Left pyelocaliceal dilatation with suggestive appearance for pyelocaliceal junction stenosis;

Renal Scintigraphy: single-functioning kidney with distension and pyelocaliceal stasis by pyelocaliceal junction stenosis (Figure 3,Figure 4).

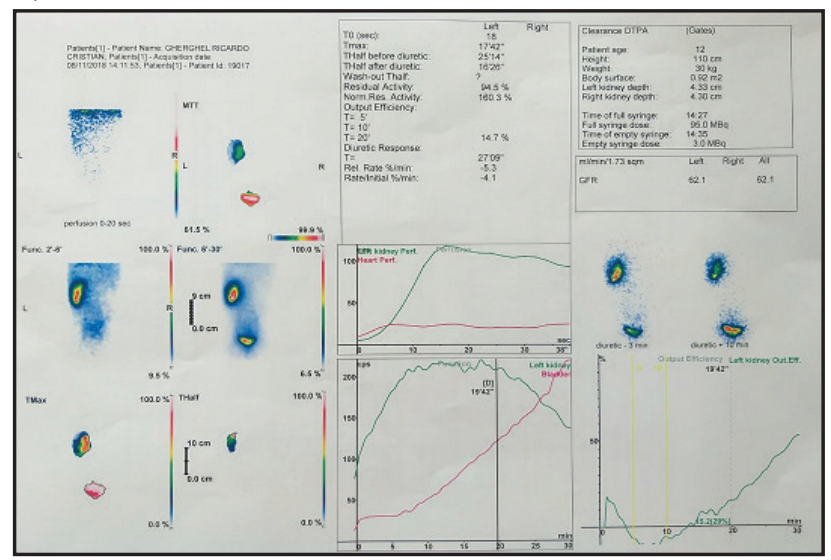

Figure 3. Renal Scintigraphy

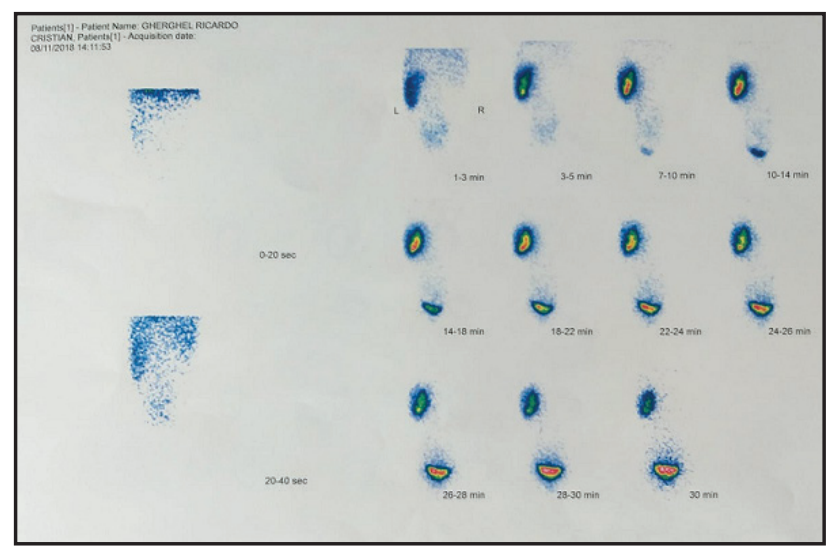

Figure 4. Renal Scintigraphy

\section{Positive diagnosis:}

Stage III left hydronephrosis as a result of pyeloureteral junction stenosis;

Non-functional hypoplastic right kidney.

Surgical treatment is neccesary, AndersonHynes pyeloplasty (dismembered pyeloplasty) is performed with Cook cathether ( 5 french cone tip), $\mathrm{CH} 4.7 / 20 \mathrm{~cm}$, lumbar drainage system. Favorable outcome, minimum drainage, on the 9th day postoperatory- the surgical drain is removed, and after 6 weeks the Cook cathether is cystoscopically extracted.

The subsequent evolution was favorable.

\section{Discussions and the particularity of the case}

We reported a case of nephrotic syndrome in a child presenting undiagnosed hydronephrosis and unilateral renal agenesis. A 3 years and 9 months old male patient presenting features of nephrotic syndrome, showed left hydronephrosis and unilateral renal agenesis.

Hydronephrosis is usually caused by a blockage of the urinary tract, in which one or both kidneys can become swollen and stretched as a result of urine build-up. It can affect people of all ages and it is sometimes seen in unborn babies during routine pregnancy check-ups (7) well-known as antenatal hydronephrosis- it does not generally cause any long-term conditions if it is diagnosed and treated promptly. If it is left untreated, the kidneys can become scarred, which can lead to kidney failure (loss of kidney function).

Hydronephrosis is usually diagnosed using an ultrasound scan (7). Further tests may be needed in order to find the cause of this condition.

Unilateral renal agenesis (URA) is defined as congenital birth defect resulting an absence of the one-side renal tissue due to an abnormality of the embryonic kidney formation $(1,2)$. Renal development starts at the 5th gestational week. There are strong interactions between the outgrowing ureteric bud of the mesonephric duct and the metanephric mesenchyme (8).

Renal agenesis can occur when the ureteric bud fails to form the ureter, the renal pelvis and the collecting ducts and the renal mesenchyme to form nephrons (9).

We have to differentiate URA from 
incomplete or abnormal renal development leading to a non-functioning kidney, like renal aplasia or multicystic dysplasia (5). However, we must note that the URA diagnosis can derive from the spontaneous involution of the multicystic dysplastic kidney (5).

URAmaybeoftenassociatedwithcongenital malformations of the contralateral kidney and/or urinary tract, like the vesicouretereal reflux and the pelviureteric junction obstruction. In addition, URA patients frequently have extra-renal anomalies, such as cardiac, gastrointestinal or genital malformations (3-5). Even though studies confirm the association of URA with congenital malformations of the kidney and the urinary tract and other anomalies, the predominance of these malformations are not well-demonstrated.

Childhood nephrotic syndromes are most commonly caused by one of two idiopathic diseases: minimal-change nephrotic syndrome and focal segmental glomerulosclerosis. The cause of idiopathic nephrotic syndrome remains unknown, but evidence suggests that it may be a primary T-cell disorder that leads to glomerular podocyte dysfunction (6). Genetic studies in children with familial nephrotic syndrome have identified mutations in genes that encode important podocyte proteins. Patients with idiopathic nephrotic syndrome are initially treated with steroids. Corticosteroidresponsiveness is of greater prognostic use than renal histology (6).

The presented patient had an excellent response to the steroid therapy and no relapses.

Renal biopsy specimen was not performed and it hasn't relapsed and hence is more likely to have minimal change disease.

Nephrotic syndrome in association with congenital anomalies like hydronephrosis and unilateral renal agenesis is extremely rare. The importance of this unusual combination was discussed respecting the literature.

\section{References}

1. Robson WL, Leung AK, Rogers RC. Unilateral renal agenesis. Adv Pediatr. 1995;42:575-92.

2. Woolf AS, Hillman KA. Unilateral renal agenesis and the congenital solitary functioning kidney: developmental, genetic and clinical perspectives. BJU Int. 2007 Jan;99(1):17-21.

3. Kaneyama K, Yamataka A, Satake S, Yanai T, Lane GJ, Kaneko K, et al. Associated urologic anomalies in children with solitary kidney. J Pediatr Surg. 2004;39(1):85-7.

4. Enomoto Y, Dohi K, Shiiki H, Minami $\mathrm{S}$, Fujii K, Ishikawa $\mathrm{H}$, et al. [Two cases of nephrotic syndrome associated with hydronephrosis]. Nihon Jinzo Gakkai Shi. 1991 Apr;33(4):431-6.

5. Westland R, Schreuder MF, Ket JCF, van Wijk JAE. Unilateral renal agenesis: a systematic review on associated anomalies and renal injury. Nephrol Dial Transplant. 2013;28(7):1844-55.

6. Eddy AA, Symons JM. Nephrotic syndrome in childhood. Lancet. 2003;362(9384):62939.

7. NHS. Diagnosis Hydronephrosis 2018 [updated 11 July 2018; cited 2020. Available from: https://www.nhs.uk/conditions/ hydronephrosis/diagnosis/.

8. Schedl A. Renal abnormalities and their developmental origin. Nat Rev Genet. 2007 Oct;8(10):791-802.

9. Kerecuk L, Schreuder MF, Woolf AS. Renal tract malformations: perspectives for nephrologists. Nat Clin Pract Nephrol. 2008 Jun;4(6):312-25. 